\title{
Bildung ist nur ein Königsweg
}

Anmerkungen zu Die Dritte Aufklärung von Michael Hampe

Dirk Jörke

\section{(2) OpenEdition \\ Journals}

Electronic version

URL: http://journals.openedition.org/ejpap/2177

DOI: 10.4000/ejpap.2177

ISSN: 2036-4091

Publisher

Associazione Pragma

Electronic reference

Dirk Jörke, « Bildung ist nur ein Königsweg », European Journal of Pragmatism and American Philosophy [Online], XII-2 | 2020, Online since 14 December 2020, connection on 15 December 2020. URL : http:// journals.openedition.org/ejpap/2177 ; DOI : https://doi.org/10.4000/ejpap.2177

This text was automatically generated on 15 décembre 2020 .

\section{(c)}

Author retains copyright and grants the European Journal of Pragmatism and American Philosophy right of first publication with the work simultaneously licensed under a Creative Commons AttributionNonCommercial-NoDerivatives 4.0 International License. 


\title{
Bildung ist nur ein Königsweg
}

\author{
Anmerkungen zu Die Dritte Aufklärung von Michael Hampe
}

\author{
Dirk Jörke
}

1 Die politischen Entwicklungen der vergangenen Jahre und hier insbesondere der Aufstieg politischer Parteien, die man gemeinhin als "populistisch" bezeichnet, aber auch der Brexit und die Wahl von Donald Trump haben in der öffentlichen Debatte für viel Unruhe gesorgt. Dabei ist eine Strategie der Moralisierung vorherrschend. Populistische Politikerinnen und Politiker, aber auch deren Wählerinnen und Wähler werden als rassistisch, sexistisch, nationalistisch und politisch nicht zurechnungsfähig gebrandmarkt. Das mag zwar aus Sicht eines politischen Liberalismus durchaus zutreffend sein, doch damit einher gehen mindestens zwei Probleme. Erstens führt die Moralisierung zu einer trügerischen Selbstzufriedenheit, auf der moralisch richtigen Seite zu stehen. In der Konsequenz braucht man sich nicht mehr um die Ursachen des gegenwärtigen Aufbegehrens vor allem der Angehörigen der unteren Mittelschicht zu kümmern. Der zweite Effekt besteht in einer doppelten Bestätigung der populistischen Gegner. Zum einen wird die populistische wir/ihr-Dichotomie dupliziert. Der populistischen Gegenüberstellung zwischen den korrupten Eliten und dem wahren oder reinen Volk wird mit der Gegenüberstellung von den guten, weltoffenen Demokraten und den bösen Rassisten geantwortet. Zum anderen führt diese Strategie nicht dahin, dass die Gräben zwischen den Lebenswelten und Weltdeutungen zwischen den zwei Lagern geringer werden oder dass die bösen Populisten auf die gute Seite konvertieren. Vielmehr dürfte diese Strategie auf Seiten der als "böse" diffamierten zu einer Bestätigung ihres Bildes von den abgehobenen und überheblichen Eliten führen. Anders ausgedrückt: Menschen, die moralisch nach unten gedrückt werden, reagieren eher selten mit Einsicht. In der Konsequenz verstärkt sich der Populismus.

2 Michael Hampe vermeidet in seinem Buch Die Dritte Aufklärung eine derartige Strategie der offenen Moralisierung. Seine Antwort auf die gegenwärtige Krise der liberalen Demokratie ist mehr Bildung oder eben auch das, was er als eine Dritte Aufklärung bezeichnet. Hampes Kernthese ist, "dass wir uns gegenwärtig nicht primär in einer Krise der Demokratie befinden, sondern eine Erosion der aufgeklärten Kultur stattgefunden hat" (S. 9). Verbunden ist damit die Annahme, dass eine aufgeklärte 
Kultur nicht notwendig an eine demokratische Gesellschaft gebunden ist. Zudem betont Hampe, dass "dasselbe politische System sowohl in einer aufgeklärten als auch in einer unaufgeklärten Kultur realisiert werden kann" (S. 9). Es ist nicht völlig klar, was aus diesen Setzungen folgt. Mein Eindruck ist allerdings, dass hiermit eine Kritik demokratischer Verfahren ohne aufgeklärte Kultur ("Die NSDAP und Hitler wurden im Dritten Reich in Wahlen bestätigt" (S. 9)) verbunden ist und dass im Zweifelsfall eine aufgeklärte Kultur ohne Demokratie einem demokratischen System ohne aufgeklärte Kultur vorzuziehen sei. Wie gesagt, so ganz offen wird dieser Punkt nicht ausgesprochen, aber auch die folgenden Überlegungen legen diese Lesart näher, etwa wenn Hampe schreibt, dass "der Verlust einer aufgeklärten Kultur gravierender ist als der der Demokratie" (S.10). Die aus meiner Sicht problematische und gänzlich unpragmatistische Pointe der Argumentation von Hampe, die durch die These des Vorrangs der Kultur gedeckt wird, besteht darin, wenn schon nicht unumwunden, so aber doch in den praktischen, d.h. realen Konsequenzen für eine neue Elitenherrschaft zu plädieren. Das trifft in der Konsequenz auch dann zu, wenn er später im Buch "nicht die Verehrung von Bildungseliten, sondern die Achtung der betreffenden Praktiken" (S. 65) empfiehlt.

3 Hampe schließt sich in seinem Buch einer weitverbreiteten Deutung der Ursachen des Populismus an. Die Wählerinnen und Wähler von Trump, Orban, Salvini und Co. seien von Unwahrheiten verführt worden. Besonderes Gewicht - und auch hier in Übereinstimmung mit einem Großteil der veröffentlichten Meinung - misst er dabei den digitalen Medien und den sogenannten "bots" also durch Algorithmen bewirkten Weiterleitungen von "likes," Hasskommentaren und Falschmeldungen bei (S. 16 f.). Diese Phänomene, die in den Sozialwissenschaften inzwischen auch gut erforscht sind (de Saint Victor 2015; Nagele 2018), haben sicherlich mit zum Wahlerfolg von Trump in den USA, der AfD in Deutschland, Marine le Pen in Frankreich oder auch zur Entscheidung über den Brexit beigetragen. Doch mit dieser Deutung sind zumindest zwei Probleme verbunden. Zum einen verstellt sie, wie auch die Antwort der Moralisierung, den Blick auf tieferliegende Ursachen der populistischen Erfolge bei Wahlen und bei der Etablierung einer hauptsächlich internetbasierten Gegenöffentlichkeit. Zum anderen knüpft diese Sicht der Dinge an eine antidemokratische Denktradition an, die sich bis auf Platon zurückführen lässt. Ich werde im Folgenden kurz auf den ersten Aspekt eingehen, um daran anschließend mich etwas ausführlicher den antidemokratischen Prämissen und Konsequenzen zu widmen.

Das zentrale Argument hinter dem Narrativ, die Anhängerinnen und Anhänger des Populismus hätten sich auf "Facebook, Instagram, Twitter und YouTube" (S. 16) von bösen Geistern verführen lassen, ist, dass sie sich irrational verhalten würden und gegen ihre wirklichen Interessen abgestimmt hätten. Aber ist das wirklich der Fall? Nehmen wir das Beispiel West Virginia. Dort lag die Zustimmung für Trump bei der Präsidentschaftswahl 2016 bei fast 70 Prozent, in einigen Gegenden des Staates gar bei nahezu 80 Prozent. Damit hat er dort sein bestes Ergebnis geholt. Waren dort besonders viele "bots" aktiv, haben wir es in West Virginia mit einem stark überdurchschnittlichen Konsum digitaler Medien zu tun? Vielleicht. Entscheidender scheint aber der Umstand zu sein, dass es sich um einen Bundesstaat handelt, der nicht nur zu den ärmsten in den USA gehört, sondern früher stark vom Bergbau geprägt war. Und auch heute noch spielt der Bergbau eine wichtige Rolle. Die Arbeiter, die in dieser Industrie noch beschäftig sind, fürchten aus nachvollziehbaren Gründen um ihre Zukunft, gilt doch die Gewinnung und Verbrennung von fossilen Energieträgern als 
einer der Hauptverursacher des Klimawandels. Hillary Clinton hat sich in ihrem Wahlkampf stark zu einer grünen Energiewende bekannt. Trump dagegen hat den menschlich verursachten Klimawandel geleugnet und sich vehement zur Fortsetzung, ja sogar zum Ausbau des Bergbaus bekannt.

5 Nun muss man als Arbeiter im Bergbau persönlich gar nicht die Meinung teilen, dass es keinen oder zumindest keinen menschlich verursachten Klimawandel gibt, um sich an den Wahlautomaten für Trump zu entscheiden. Dafür reicht allein die nicht gänzlich unberechtigte Sorge um die individuelle Zukunft oder auch die Zukunft der Familie. Es ist zwar zu erwarten, dass diese Person Posts, die den Klimawandel leugnen, "liken" wird, doch hätte er auch, wenn es diese nicht gäbe, mit einer hohen Wahrscheinlichkeit Trump gewählt.

$6 \quad$ Ein weiteres Beispiel: die Entscheidung der Mehrheit der britischen Bürgerinnen und Bürger, die Europäische Union zu verlassen. Auch hier haben Kommentatoren zu Recht darauf verwiesen, dass mit falschen Zahlen Stimmung gemacht worden sei. Doch sollte man bezweifeln, dass ohne all diese Fehlinformationen das Ergebnis anders ausgefallen wäre. Dafür spricht zunächst die Tatsache, dass bei der letzten Wahl im Vereinigten Königreich Boris Johnson, der sich für einen klaren Brexit ausgesprochen hat, mit sehr großer Mehrheit bestätigt worden ist. Und dies gut drei Jahre nachdem die Briten sich mit knapper Mehrheit gegen einen Verbleib in der Europäischen Union ausgesprochen haben. Eigentlich wäre damit genügend Zeit gewesen, um die Brexitbefürworter über ihre Irrtümer aufzuklären. Und das wurde auch intensiv versucht - allein, es hat nicht zum gewünschten Ergebnis geführt. Ein Grund dafür mag nun in der Tat sein, dass viele Menschen keinen Zugang mehr zu "rationalen" Argumenten und Fakten haben, sondern sich in ihre "Filterblasen" eingenistet haben. Doch diese Erklärung greift wie bereits bei der Wahl von Trump zu kurz. Denn sie verstellt erneut den Blick auf die Interessen und durchaus auch zweckrationalen Motive, die Menschen dazu bewogen haben, für einen Austritt aus der EU zu stimmen. Ein wesentliches Thema bei der Brexitkampagne war die Frage der Migration. Das Recht auf Freizügigkeit gehört zu den vier Grundfreiheiten der Europäischen Union und hat damit gleichsam Verfassungsrang. Von den Migrationsbewegungen der letzten Jahre waren jedoch die Menschen im Vereinigten Königreich höchst unterschiedlich betroffen. Über das genaue Ausmaß dieser Betroffenheit lässt sich freilich streiten, doch ist es schwer zu bezweifeln, dass Menschen aus der "nichtakademischen Mittelklasse" und der "neuen Unterklasse" im Unterschied zu den Angehörigen der "Akademikerklasse" nicht unbedingt zu den Gewinnern der Einwanderung gehörten (Reckwitz 2017). Die Abstimmung über den Brexit spiegelt diese Interessenlage. In den sozial abgehängten Gebieten im Norden Englands stimmte die Mehrheit für den Austritt, in den Universitätsstädten wie Oxford und Cambridge und auch in den besseren Lagen von London sprachen sich die meisten Bürgerinnen und Bürger für einen Verbleib aus.

7 Eine ganz ähnliche Analyse lässt sich über das Wahlverhalten in Frankreich oder auch in Deutschland durchführen. Auch dort zeigt sich, dass die Zustimmung für populistische Parteien gerade bei den Angehörigen der unteren Mittelklasse, also Menschen, die zumeist nicht über höhere Schulabschlüsse verfügen, besonders hoch ist. Und hinter diesem Wahlverhalten stehen reale Ängste und Erfahrungen. Diese müssen nicht nur ökonomisch bedingt sein, sondern sind oftmals auch Resultate mannigfaltiger Missachtungserfahrungen, etwa im Arbeitsleben, das für diese Menschen selten durch Kreativität und Selbstbestimmung geprägt ist, oder aber auch 
durch die mediale Abwertung von Lebensstilen, etwa den des "alten weißen Mannes" oder die "Typen, die Zigaretten rauchen und mit Diesel fahren," wie Benjamin Griveaux, der inzwischen zurückgetretene ehemalige Pressesprecher von Emanuel Macron, die Gelbwesten bezeichnet hat. ${ }^{1}$ Das hinterlässt Spuren und führt in einer vielleicht nachvollziehbaren Weise zu dem Wunsch, es den Eliten heimzuzahlen.

Die Fokussierung auf die vermeintliche Manipulation der Anhänger des Populismus durch böse Mächte blendet diese Zusammenhänge jedoch nicht nur aus, sie hat selbst einen verschwörungstheoretischen Zug; auch hier lässt sich eine Parallele zu populistischen Narrativen sehen. Eine soziologisch aufgeklärtere und im Sinne des Fortbestands der liberalen Demokratie produktivere Sicht der Dinge würde sich demgegenüber auf weitere und vielleicht zentralere Ursachen der gesellschaftlichen Polarisierung konzentrieren, also etwa danach zu fragen, weshalb in den lukrativen Innenstadtlagen der Metropolen der Stimmenanteil der populistischen Parteien erheblich geringer ausfällt als in den städtischen Peripherien oder jenseits der urbanen Zentren.

9 Hampes Auffassung, die Unterstützer des Populismus seien durch eine moderne Form der Demagogie manipuliert worden, erinnert an Platons Demokratiekritik. Platon hat sich bekanntlich in vielen Dialogen und am deutlichsten in der Politeia sehr kritisch mit der damaligen radikaldemokratischen Praxis in Athen auseinandergesetzt. Typisch ist etwa folgende Passage:

Wenn sie in dichter Masse beisammen sitzen in den Volksversammlungen oder Gerichtshöfen oder Theatern oder im Kriegslager oder bei sonstigen Ansammlungen einer großen Menge in öffentlichen Angelegenheiten und durch starken Lärm je nachdem ihren Tadel oder ihr Lob über das Vorgetragene kundgeben, beides in ganz übertriebener Weise, mit Schreien und Klatschen, und sich ihnen selbst noch die Felsen und die ganze Örtlichkeit, wo sie tagen, mit ihrem Widerhall zugesellen, so daß der Lärm für Tadel und Lob auf das Doppelte verstärkt wird. $(492 \mathrm{~b} / \mathrm{c})$

10 Hier zeichnet Platon ein sehr suggestives Bild der vermeintlichen Dynamiken von Versammlungen. Beim Lesen dieser Zeilen spürt man geradezu das Geschrei und den Tumult in der Ekklesia und auf der Agora. Es werden Bilder und Emotionen beim Leser wachgerufen, die unangenehm sind und ihren Effekt nicht verfehlen dürften. So entsteht das Bild, dass überall dort, wo das Volk in "dichter Masse" zusammenkommt und über Fragen des Gemeinwohls diskutiert, Leidenschaften auf der einen und Demagogie auf der anderen Seite das Geschehen bestimmen. Eine vernünftige Entscheidungsfindung, die auf das Gute zielt, ist unter diesen Umständen völlig undenkbar. Platon wollte die Irrationalität der Massen durch die Weisheit der Philosophen einschränken; eine Lösung, die in der demokratisch geprägten Kultur Athens auf wenig Begeisterung gestoßen wäre, was Platon auch antizipiert hat. Der Philosoph, der in die Höhle zurücksteigt, wird erschlagen.

11 Was in Athen die Ekklesia oder der Marktplatz war, sind heute die digitalen Räume. Auch dort kommt eine "dichte Masse" zusammen, zwar nicht in einem körperlichen Sinne, aber doch so, dass in diesen artifiziellen Sphären eine ganz ähnliche Dynamik zu beobachten sei. Hampe spricht von einem "kollektive[n] Sturz in den Vertrauensverlust gegenüber beliebigen Eliten [...] gepaart mit einer kaum zu befriedigenden Lust am leeren Gerede, an Bullshit" (S. 32). Das hätte Platon nicht bissiger formulieren können. Auch mit Blick auf die Therapie folgt Hampe dem antiken Vorbild. Zwar plädiert Hampe nicht für eine Philosophenherrschaft, dennoch findet sich bei ihm die 
platonische Entgegensetzung von bloßer Meinung und wahrem Wissen, von doxa und episteme: "Man kann Meinungen mit den hauchdünnen Plastiktüten vergleichen, die kostenlos in einigen Supermärkten aus einem Kästchen für die Verpackung des Obsteinkaufs erhältlich sind. Wissen gleicht dagegen sorgfältig gefertigten RindslederReisetaschen." (S. 34 f.).

Aus pragmatistischer Sicht ist dieses Bild in zweifacher Hinsicht irritierend. Zunächst einmal wird hier ein Dualismus konstruiert, der Dewey zufolge charakteristisch für die platonische Suche nach Gewissheit ist. Einer unreinen, weil wandelbaren Sphäre des bloßen Meinens wird die reine und unveränderliche Sphäre des Wissens entgegengestellt. Um im Bild zu bleiben: die Plastiktüten halten mit Glück gerade bis zum heimischen Kühlschrank, die Rindsleder-Tasche, wenn auch nicht für die Ewigkeit, dann doch zumindest "das ganze Leben" (S. 35) lang. Diese Übernahme einer zutiefst platonischen Denkfigur durch Hampe ist umso überraschender, als er sich ja selbst gegen Ende des Buches ganz explizit auf Deweys naturalistische Metaphysik, wie er sie in Erfahrung und Natur (Dewey 1995) entwickelt hat, beruft und der zufolge gerade "die Mehrdeutigkeiten, die ungewissen Möglichkeiten und Zufälle" (S. 77) für die menschliche Welterfahrung charakteristisch sind. Eine derartige Metaphysik verträgt sich aber nicht mit der dualistischen Gegenüberstellung von doxa und episteme und im letzten Abschnitt seines Buches wird diese Denkfigur von Hampe auch konsequenterweise nicht mehr bemüht. Stattdessen betont er völlig zu Recht die Offenheit des Wissens. Hier sehe ich zumindest eine Spannung zwischen stärker platonischen Zügen, die, aber hier kann ich nur spekulieren, seiner Wahrnehmung der aktuellen massendemokratischen Unkultur geschuldet sein mögen, und einer soliden pragmatistischen Argumentation. Nur beiläufig erwähnt: Eigentlich bin ich ganz froh, dass ich die von mir erworbenen Erdbeeren in einer dünnen Plastiktüte oder auch Papiertüte transportieren kann.

13 Die strikte Entgegensetzung von doxa und episteme ist auch aus einem zweiten Grund aus pragmatistischer Sicht zurückzuweisen. Denn sie dient der Rechtfertigung der Herrschaft der Wissenden, also derjenigen, die sich nicht durch Demagogen aufwiegeln oder von flüchtigen Moden hinters Licht führen lassen. Wie bereits erwähnt, plädiert Hampe nicht für eine Philosophenherrschaft oder auch eine Herrschaft der Experten, die sich gegenwärtig in der politischen Theorie einer gewissen Beliebtheit erfreut. ${ }^{2}$ Hampe erhofft sich dagegen durch Bildung, durch "Wahrheitspraktiken, Praktiken des Lesens, des Überzeugens und Argumentierens" (S. 65) eine Rettung der Kultur und damit auch der liberalen Demokratie. Dabei kann er sich auf den ersten Blick durchaus auf die pragmatistische Tradition und insbesondere auf John Dewey stützen, auch wenn der US-amerikanische Philosoph in diesem Zusammenhang nicht explizit erwähnt wird. Die Bedeutung der Erziehung kann in Deweys Werk gar nicht hoch genug geschätzt werden. Das übergreifende Ziel seines Philosophierens war eine Erziehung zur Demokratie, Demokratie nicht nur in einem engen politisch-institutionellen, sondern in einem viel umfassenderen nahezu gesamtgesellschaftlichen Sinne verstanden. Und dafür war auch bei Dewey Bildung von entscheidender Bedeutung, wobei er stärker, als es bei Hampe der Fall ist, auch die praktisch-technische Ausbildung der Schülerinnen und Schüler betonte.

14 Auch in einem weiteren Sinne decken sich die Überlegungen von Dewey und Hampe, und zwar heben beide die Praktiken des Argumentierens und Überzeugens bei der Lösung politischer Probleme hervor. So schreibt Dewey in Die Öffentlichkeit und ihre 
Probleme: "Das wesentliche Erfordernis besteht [...] in der Verbesserung der Methoden und Bedingungen des Debattierens, Diskutierens und Überzeugens. Das ist das Problem der Öffentlichkeit." (Dewey 1996: 173). In der Tat lässt sich bei Dewey, und insbesondere in Die Öffentlichkeit und ihre Probleme, eine Theorie deliberativer Demokratie finden. Zudem ging es Dewey auch um eine Demokratisierung der Expertise. Darunter verstand er zum einen die Verbreitung insbesondere sozialwissenschaftlicher Erkenntnisse in der Bevölkerung, etwa über entsprechende Zeitungen und Radiosendungen. Zum anderen hat Dewey sich zeitlebens auch für die Übertragung naturwissenschaftlicher Methoden auf moralische, soziale und politischen Problemsituationen ausgesprochen. Allerdings hat sich Dewey mit diesen Forderungen nicht gänzlich $\mathrm{zu}$ Unrecht den Vorwurf der Wissenschaftsgläubigkeit und der Machtblindheit eingehandelt (Mills 1964). Und auch aus heutiger Perspektive erscheint die Forderung, Phänomene wie Populismus und die zunehmende Abschottung sozialer Milieus allein durch mehr Bildung und mehr Deliberation überwinden zu wollen, wenig überzeugend.

Denn in gewisser Weise handelt es sich dabei um etwas, was man im Anschluss an Dewey als "intellektualistischen Fehlschluss" (Dewey 1998: 220) bezeichnet könnte. Der "intellektualistische Fehlschluss" führt zu einer "Herabwürdigung der Dinge, die wir durch Liebe, Begierde Hoffnung, Furcht und Absicht" (ebd.) erfahren. Philosophen, die sich hauptsächlich mit kognitiven Erkenntnissen und Bildungsgütern auseinandersetzen, glauben in ihrem privilegierten Zugang zur Welt, die Lösung gesamtgesellschaftlicher Probleme finden zu können.

16 Warum ist diese bei "Philosophen, Soziologen und überhaupt alle[n], die das Nachdenken über die Welt professionell betreiben" verbreitete "scholastische Sicht" (Bourdieu 1998: 205) problematisch? Die Antwort, die man mit Dewey und Pierre Bourdieu geben kann, ist, dass sie ihren notwendig partiellen Standpunkt verallgemeinern, dadurch aber einen großen Bereich der Wirklichkeit ausklammern. Um das an einem Beispiel zu illustrieren, möchte ich kurz auf ein zunächst sehr irritierendes Phänomen hinweisen, nämlich die gerade in den französischen Banlieues häufig zu beobachten Verwüstungen von Bibliotheken, also der Orte, von denen sich Hampe eine Überwindung der gegenwärtigen Kultur- und Demokratiekrise erwartet. ${ }^{3}$ Doch weshalb kommt es zu dieser aus unserer Sicht schwer nachvollziehbaren Gewalt gegen Einrichtungen, die den Bewohnerinnen und Bewohnern der Banlieues doch eigentlich einen sozialen Aufstieg ermöglichen sollten? Zum einen, weil viele von ihnen ahnen, dass es sich um ein leeres Versprechen handelt, denn im Bereich der Bildung verfügen sie über schlechtere Startchancen, gerade in einem Land wie Frankreich, dass von einer hohen Bildungsexklusivität geprägt ist. Zum anderen, und vielleicht noch wichtiger, stehen Bibliotheken für eine Welt, die ihnen verhasst ist, nämlich die Welt der Bessergestellten, zu denen sie, zumindest in ihrer Wahrnehmung, niemals aufschließen können. Anders ausgedrückt, viele Menschen in diesen Vierteln wird man nicht über mehr Bildung erreichen können. Das Gleiche, wenn vielleicht auch in einem etwas schwächeren Umfang, dürfte auch auf jene Menschen zutreffen, die zu den Unterstützern populistischer Parteien gehören. Sie dürften diejenigen, die ihnen sagen, sie sollten sich mehr bilden, dann würden sie sowohl einen anderen Blick auf die Realität entwickeln als auch größere Chancen auf dem Arbeitsmarkt bekommen, ähnlich begegnen, wie die Höhlenbewohner dem Philosophen. Und das nicht zuletzt deshalb, weil zumindest aus ihrer Sicht das bedeutet, so zu werden, wie jene Menschen, mit denen sie kaum noch über gemeinsame Erfahrungsräume verfügen, sich also deren 
Werten anzupassen. Zumindest kurzfristig dürfte also die alleinige Forderung nach mehr Bildung oder einer Dritte[n] Aufklärung für viele Menschen daher nicht allzu verheißungsvoll sein. Vielmehr dürfte sie als ein weiterer Beleg für die vermeintliche Abgehobenheit der Eliten wahrgenommen werden.

Michael Hampe würde die hier skizzierten Einwände sicherlich weit von sich weisen. Und ich möchte auch nicht behaupten, dass die geschilderten Implikationen seines Vorschlages von ihm intendiert sind. Wie so viele macht auch er sich völlig zu Recht Sorgen über den momentanen Zustand der westlichen Demokratien. Es sollte aber deutlich geworden sein, dass ich eine andere Deutung der Ursachen der gegenwärtigen Krisentendenzen im Allgemeinen und der populistischen Revolte im Besonderen habe. Diese haben sicherlich auch viel mit Kultur und mangelnder Bildung zu tun. Aber gewiss nicht nur und vielleicht auch nicht in erster Linie. Wie könnte eine überzeugendere Antwort auf die Demokratie- und Kulturkrise aussehen?

Hier lohnt eine erneute Hinwendung zu John Deweys Sozialphilosophie, und zwar in zweifacher Hinsicht. Zum einen hat er bei aller Betonung der Notwendigkeit von Erziehung und Bildung immer auch die Relevanz nicht-kognitiver Erfahrungen hervorgehoben. Zum anderen, und für die Lösung der gegenwärtigen Demokratiekrise noch wichtiger, ist Dewey in seiner politischen Theorie nicht bei der Forderung nach besseren Methoden des Debattierens stehengeblieben. Vor dem Hintergrund der zunehmenden sozialen Spaltung der US-amerikanischen Gesellschaft hat, insbesondere nach dem Ausbruch der Weltwirtschaftskrise, sich Dewey zu einem demokratischen Sozialisten entwickelt, der die damaligen Krisensymptome auf die sehr ungleichen Eigentumsverhältnisse in der US-amerikanischen Gesellschaft zurückführte. Um dem zu begegnen, hat sich Dewey nicht nur ganz konkret für die Sozialisierung des Bodens, der Banken und der Schlüsselindustrien ausgesprochen, sondern in seinen stärker theoretischen Schriften wie Individualism. Old and New (1930) und Liberalism and Social Action (1935) auch ein neues Verständnis von Freiheit entwickelt, das es erlaubt, mit demokratischen Mehrheiten in vermeintliche Eigentumsrechte einzugreifen (vgl. Jörke 2020).

\section{BIBLIOGRAPHY}

BOURDIEU Pierre, (1998), Praktische Vernunft, Frankfurt-a.-Main, Suhrkamp.

BRENNAN Jason, (2017), Gegen Demokratie: Warum wir die Politik nicht den Unvernünftigen überlassen dürfen, Berlin, Ullstein.

DEWEY John, (1930/1984b), Individualism Old and New, in Jo Ann Boydston (Hrsg.), The Later Works, Vol. 5, Carbondale, Southern Illinois University Press, 41-143.

DEWEY John, (1935/1987a), Liberalism and Social Action, in Jo Ann Boydston (Hrsg.), The Later Works, Vol. 11, Carbondale, Southern Illinois University Press, 1-65.

DEWEY John, (1995), Erfahrung und Natur, Frankfurt-a.-Main, Suhrkamp. 
DEWEY John, (1996), Die Öffentlichkeit und ihre Probleme, Bodenheim, philo.

DEWEY John, (1998), Die Suche nach Gewißheit, Frankfurt-a.-Main, Suhrkamp.

JÖRKE Dirk, (2020), “John Deweys demokratischer Sozialismus," in Hauke Brunkhorst, Felix

Petersen \& Martin Seelinger (Hrsg.), Pragmatistische Sozialforschung, Stuttgart, Metzler (i.E.).

MILLS C. Wright, (1969), Sociology and Pragmatism, The Higher Learning in America, New York, Oxford University Press.

NAGELE Angela, (2018), Die digitale Gegenrevolution. Online-Kulturkämpfe der Neuen Rechten von 4chan und Tumblr bis zu Alt-Right und Trump, Bielefeld, transcript.

PLATON, (1988), Der Staat, übersetzt und erläutert von Otto Appelt, Hamburg, Meiner.

RECKWITZ Andreas, (2017), Die Gesellschaft der Singularitäten, Berlin, Suhrkamp.

ROSANVALlON Pierre, (2010), Demokratische Legitimität. Unparteilichkeit - Reflexivität - Nähe, Hamburg, Hamburger Edition.

DE SAINT VICTOR Jacques, (2015), Die Antipolitischen, Hamburg, Hamburger Edition.

WILLKE Helmut, (2016), Dezentrierte Demokratie: Prolegomena zur Revision politischer Steuerung, Berlin, Suhrkamp.

\section{NOTES}

1. Vgl. Le Figaro, verfügbar unter: [https://www.lefigaro.fr/vox/monde/ 2018/10/29/31002-20181029ARTFIG00214-le-mepris-siderant-de-griveaux-pour-les-gars-quifument-des-clopes-et-roulent-au-diesel.php] (letzter Aufruf 23 Juni 2020).

2. Etwa Brennan (2017), Willke (2016), Rosanvallon (2010), wobei letzterer sich besonders bemüht, Expertengremien als demokratisch zu legitimieren.

3. So wurden zwischen 1996 und 2015 in den Banlieues 72 Bibliotheken zerstört; vgl. [https:// www.sueddeutsche.de/kultur/paris-bibliothek-verwuestet-1.2440729]; ein lesenswerter Essay zu diesem Phänomen findet sich hier: [https://booksandideas.net/Why-are-people-setting-fireto.html].

\section{AUTHOR}

\section{DIRK JÖRKE}

Technische Universität Darmstadt

joerke[at]pg.tu-darmstadt.de 